\title{
Novel D-hordein-like HMW Glutenin Sequences Isolated from Psathyrostachys juncea by Thermal Asymmetric Interlaced PCR
}

\author{
S.F. DAI ${ }^{1 * *}$, X.F. XUE ${ }^{1 * *}$, Y.F. $\mathrm{WANG}^{1}$, Y.L. XIE ${ }^{1}$, Z.P. SonG ${ }^{1}$, D.Y. XU' \\ Z.J. WEN ${ }^{1}$ and Z.H. YAN ${ }^{1 *}$ \\ ${ }^{1}$ Triticeae Research Institute, Sichuan Agricultural University, 611130, \\ Wenjiang District, Chengdu, Sichuan, 611130, China
}

(Received 20 June 2016; Accepted 27 June 2017;

Communicated by F. Békés)

\begin{abstract}
New high-molecular-weight glutenin (HMW glutenin) sequences isolated from six Psathyrostachys juncea accessions by thermal asymmetric interlaced PCR differ from previous sequences from this species. They showed novel modifications in all of the structural domains, with unique $\mathrm{C}$-terminal residues, and their $\mathrm{N}$-terminal lengths were the longest among the HMW glutenins reported to date. In their repetitive domains, there were three repeatable motif units: 13-residue [GYWH(/I/Y)YT(/Q)S(/T)VTSPQQ], hexapeptide (PGQGQQ), and tetrapeptide (ITVS). The 13-residue repeats were restricted to the current sequences, while the tetrapeptides were only shared by D-hordein and the current sequences. However, these sequences were not expressed as normal HMW glutenin proteins because an in-frame stop codon located in the C-termini interrupted the intact open reading frames. A phylogenetic analysis supported different origins of the $P$. juncea HMW glutenin sequences than that revealed by a previous study. The current sequences showed a close relationship with D-hordein but appeared to be more primitive.
\end{abstract}

Keywords: Psathyrostachys juncea, HMW glutenin, sequence analysis, thermal asymmetric interlaced PCR, phylogenetic analysis

\section{Introduction}

High-molecular-weight glutenin subunits (HMW-GSs) are important storage proteins in annual and perennial species of the tribe Triticeae (Shewry et al. 2003; Rasheed et al. 2014). Over the past 30 years, Triticeae HMW-GSs have been extensively studied because of their important roles in determining the end-use quality of wheat flours and in understanding the evolutionary relationships among species (Shewry et al. 2003; Garg et al. 2009; Rasheed et al. 2014).

Triticeae $\mathrm{x}$ - and y-type HMW-GS genes share four structural domains: the signal peptide, $\mathrm{N}$ - and $\mathrm{C}$-termini, and a larger central repetitive region flanked by the $\mathrm{N}$ - and $\mathrm{C}$ termini (Shewry et al. 2003). The central repetitive region is composed of three repeatable

\footnotetext{
*Corresponding author; E-mail: zhyan104@163.com; Phone/Fax.: +86-28-82650350

**Contributed equally to this work.
} 
units: hexa- (PGQGQQ), nona- (GYYPTSPQQ), and tri- (GQQ) peptides. The hexa- and nonapeptides are common to $\mathrm{X}$ - and $\mathrm{y}$-type subunits, whereas the tripeptide is restricted to $\mathrm{x}$-type subunits. Novel modifications in the domain structure of Triticeae HMW-GSs that differ from standard $\mathrm{x}$ - or $\mathrm{y}$-type subunits have also been reported ( $\mathrm{Gu}$ et al. 2003; Pistón et al. 2007). These modifications included variations in the $\mathrm{N}$ - and C-terminal domain lengths, nucleotide compositions, and repeatable motif units (Gu et al. 2003; Shewry et al. 2003; Pistón et al. 2007).

Wheat's wild relatives contain many novel HMW-GS alleles that are deficient in bread wheat. HMW-GSs, mostly from Triticeae genera, were molecularly characterized to identify novel HMW-GSs and to understand the evolutionary relationships within Triticeae (Garg et al. 2009). However, the HMW-GSs in a few genera, such as Psathyrostachys, are still not well characterized, except for the sequence information provided by Kong et al. (2014).

Psathyrostachys is a perennial Triticeae genus that has Ns genomes. The species in this genus are distantly related to wheat A, B, and D genomes, and to the other Triticeae species with I, H, R, St, P, E, and W genomes (Hsiao et al. 1986). Psathyrostachys species provide many valuable traits for wheat improvement (Cao et al. 2008). Psathyrostachys juncea (Russian wild rye) is an important forage crop for livestock and also carries valuable traits, including tolerance to drought and salinity, as well as resistance to barley yellow dwarf virus (Plourde et al. 1990). Successfully hybridizations between $P$. juncea and common wheat/durum wheat (Triticum turgidum L.) provide an alternative way to transfer these biotic and abiotic traits from P. juncea to wheat (Plourde et al. 1990; MujeebKazi et al. 1995).

In our previous study, different start regions of $P$. juncea HMW-GSs were found (Yang et al. 2010), which made it difficult to acquire HMW-GSs from this species using conventional PCR. Thermal asymmetric interlaced PCR (TAIL-PCR) is a known sequence-based PCR that efficiently isolates unknown DNA flanking regions (Liu and Cheng 2007). In this study, $P$. juncea HMW glutenin sequences were isolated and characterized by TAILPCR. Unexpectedly, the current sequences showed high similarities to D-hordein but were dissimilar to homologs from the same species reported by Kong et al. (2014). In the conservative $\mathrm{N}$ - and C-terminal domains, novel modifications were found in the length, nucleotide composition and central repetitive domain motif. This sequence information is important for understanding the origin of HMW-GSs in Psathyrostachys $(2 n=2 x=14$, NsNs) and Leymus $(2 n=4 x=28$, NsNsXmXm) species, which share Ns genomes, and for determining the evolutionary relationships among Triticeae HMW-GSs.

\section{Materials and Methods}

\section{Plant materials}

Nine $P$. juncea accessions (PI 499559, PI 531826, PI 75737, PI 315080, PI 429801, PI 430866, PI 565065, PI 565074 and PI 619483) were used in this study. The arbitrarily selected accessions were used for DNA extraction, HMW-GS separation and sequencing of the 5'-promoter and coding sequences of HMW-GSs. 


\section{$H M W-G S$ extraction and separation}

The HMW-GSs of four P. juncea accessions, PI 429801, PI 565074, PI 565065, and PI 531826 , were extracted and separated by sodium dodecyl sulfate polyacrylamide gel electrophoresis (SDS-PAGE) as previously described (Sun et al. 2014). Two wheat cultivars, Chinese Spring (CS) and Shinchunaga (Sh), with known HMW-GSs, were used as references to identify the $P$. juncea HMW-GSs.

\section{Isolation of HMW-GS coding regions by TAIL-PCR}

The HMW-GSs' 5'-promoter sequences were amplified using the primers PPFyE and PPRy-2P. The TAIL-PCR primers used for amplifying the unknown HMW-GSs' coding sequences were designed based on the 5'-promoter sequences (Table S1*). All TAILPCRs consisted of three consecutive PCR runs: pre-amplification PCR, primary TAILPCR, and secondary TAIL-PCR. All of the PCRs were conducted in ABI 9700 DNA cyclers (PE Company, USA), and the conditions were the same as those described in Liu and Cheng (2007). All PCR information is listed in Tables S1 and S2, and in Fig. S1. A new PCR primer pair, PPORF/PPORR, which replaced the TAIL-PCR primers for amplifying the coding sequences, was designed with a 5 '-promotor region sequence and third-round PCR products.

The PCR products were separated on $0.8 \%$ agarose gels. Target DNA fragments were recovered, ligated into $p M D 18-T$ vectors (Takara, China), and used to transform chemically competent Escherichia coli DH10B cells to acquire positive clones. At least three individual clones for each transformation experiment were sequenced to minimize sequencing errors.

Phylogenetic relationships among y-type HMW-GSs from P. juncea and other Triticeae species

Orthologous y-type HMW-GS representatives from Triticeae were used for phylogenetic tree construction. The HMW-GSs originated from 20 different Triticeae genomes. Phylogenetic trees were constructed based on the amino acid (AA) residues of the N-termini, C-termini, and both the $\mathrm{N}$ - and $\mathrm{C}$-termini using MEGA 6.0 with the complete deletion of gaps and missing data (Tamura et al. 2013). Three maximum likelihood trees were constructed and assessed using 1,000 bootstrap replicates. These y-type HMW-GSs included the following: Ay (X 03042), By9 (X 61026), Dy12 (X 03041), Cy (AF 476960), Ee1.8y (AY 298724), Fy (FJ 481573,), Gy (HM 131806), D-hordein (EF 417988), Ky (AY 834230), Oy (FJ 481569,), Py (DQ 073531), Qy (FJ 481571), Ry (AF 216869), Sty (DQ 344030), Tay (AY 303125), Uy (AF 476962), Vy (FJ 600491), Wy (JN 591653), Xey (FJ481574), Nsy (Ns1-Ns4, KF 631404, KF 631405, KF 631406, and KF 631407), Racy (KC767940) and Chiy (Chiy1-Chiy2, KC 767941, and KC 767942).

*Further details about the Electronic Supplementary Material (ESM) can be found at the end of the article. 


\section{Results}

Analysis of P. juncea $H M W-G S s$

As shown in Fig. S2, P. juncea HMW-GSs varied among and within accessions. For the seeds of each accession, two or more protein bands that exhibited similar electrophoretic mobility as subunits $2.2,2$, and 7 of the wheat references were considered $P$. juncea HMW-GSs. More than two HMW-GSs were detected in diploid P. juncea, which indicated that $P$. juncea was cross-pollinated and that the HMW-GSs of this species were heterozygous.

\section{Sequence variation of the 5'-promoter regions}

The 5'-promoter regions, including partial open reading frames (ORFs) of $\sim 210 \mathrm{bp}$, of HMW-GSs in five $P$. juncea accessions were amplified (Fig. S3a). After sequencing, we obtained five nucleotide sequences with 1,134 or 1,135 bp from five accessions. The GenBank accession numbers for these sequences are from KT 878873 to KT 878877 . The nucleotide compositions were conserved, and only limited insertion/deletions and substitutions were observed (Fig. S4). At the beginning of the ORFs, we found two bases different from those of the known HMW-GSs (position 11 downstream of ATG from G to A, resulting in GGT/Arg to AGT/Gln, and position 19 from $\mathrm{C}$ to T, resulting in $\mathrm{CTC} / \mathrm{Leu}$ to TTC/Phe) (Fig. S4).

Isolation of the full ORFs of P. juncea $H M W-G S s$ by TAIL-PCR, and a comparison with homologous sequences of other Triticeae species

A set of TAIL-PCR primers were designed to amplify the DNA fragments that contained the ORFs of HMW-GS (Tables S1 and S2, Fig. S1). During pre-amplification, some faint bands that varied from approximately $0.5-2.5 \mathrm{~kb}$ were amplified from three different P. juncea accessions (Fig. S3b). In the primary TAIL-PCR, some major bands of $\sim 2.0 \mathrm{~kb}$ were amplified using diluted pre-amplification products as templates (Fig. S3c). In the secondary TAIL-PCR, major PCR fragments of $\sim 2.0 \mathrm{~kb}$ were continually amplified using diluted primary TAIL-PCR products as templates (Fig. S3d). The larger secondary TAILPCR bands of $\sim 2.0 \mathrm{~kb}$ were cloned and sequenced, whereas other bands smaller than 2.0 $\mathrm{kb}$ were omitted.

The DNA fragments contained a 1.3-kb ORF and some 3' downstream flanking sequences. These ORFs had a unique C-terminal end, with putative AA residues AMLANK instead of the standard ALSASQ. To simplify the PCR for isolation of HMW-GSs with this type of C-terminal end, a new reverse primer, PPORR, was designed to replace the TAIL-PCR primers AC1 and AD1, and was used in combination with PPORF (Fig. S1 and Fig. S3e). Similar DNA fragments of $\sim 1.3 \mathrm{~kb}$ were amplified from different accessions. A total of six 1.3-kb ORFs, Glu-Ns1-6 were acquired from the six accessions. These sequences were deposited in the GenBank database under accession numbers KT 878870 to KT 878875. 


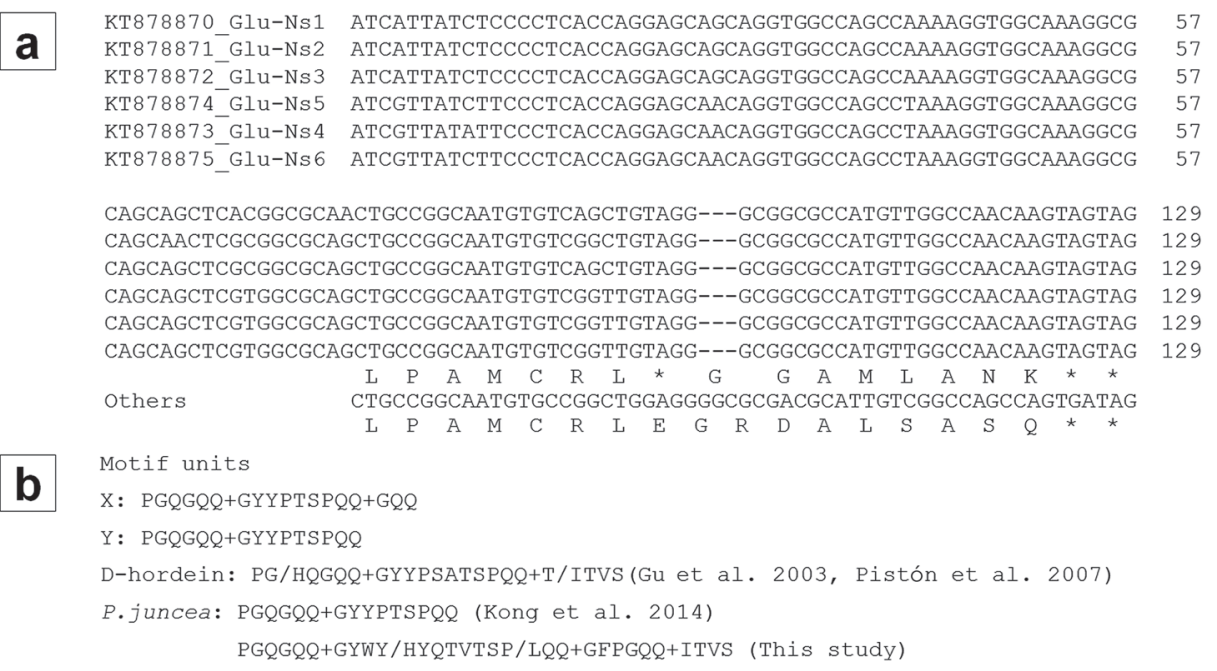

Figure 1. Comparison of HMW-GS genes from P. juncea with orthologous sequences from other Triticeae species in the C-terminus (a) and repetitive domains (b)

These ORFs were not expressed as normal HMW-GS proteins because $1-5$ in-frame stop codon(s) were located in the repetitive domain and/or C-terminals (Fig. 1a and Fig. $\mathrm{S} 5 \mathrm{a})$. Although these $\mathrm{Glu}-\mathrm{N} s$ 's were not expressed as normal proteins, novel modifications in gene structures were noted (Fig. 1 and Fig. S5). To explain these unique gene structures, the ORFs were translated to AA residues after ignoring the stop codons. In the repetitive domain, there were three motif units: 13-residue [GYWH (/I/Y) YT (/Q) S (/T) VTSPQQ], hexapeptide (consensus PGQGQQ) and tetrapeptide (ITVS). The C-termini of current Glu-Ns's are unique, especially the residues at the start and end. The normal C-terminal residues at the start and end are SPYHVSA and GGALSASQ, respectively, but they were replaced by II (V) IS (F) PHQ II (V) IS (F) PHQ and GGAMLANK in the current Glu-Ns (Fig. S5b, box). The current Glu-Ns had the longest N-terminal lengths and D-hordein had the second longest among all of the HMW-GSs, with 120 and $110 \mathrm{AA}$ residues, respectively (Fig. S5b). Large DNA fragment insertions in D-hordein and Glu$N s$ were responsible for the longer N-terminal lengths (Fig. S5b, box).

\section{$H M W-G S$ phylogenetic trees for P. juncea and other Triticeae species}

Three maximum likelihood trees for HMW-GSs of $P$. juncea and other Triticeae species were constructed (Fig. 2). The phylogenetic tree based on the AA residues of both the Nand C-termini supported different origins of the Glu-Ns compared with that previously reported for P. juncea (Kong et al. 2014) and with those in our study (Fig. 2a). All of the HMW-GSs formed two main branches, which corresponded to the Glu-Ns previously reported from $P$. juncea (Kong et al. 2014) and those in our study. The current Glu-Ns clustered together, forming a clade of their own (Fig. 2a, long arrows), and revealed a 

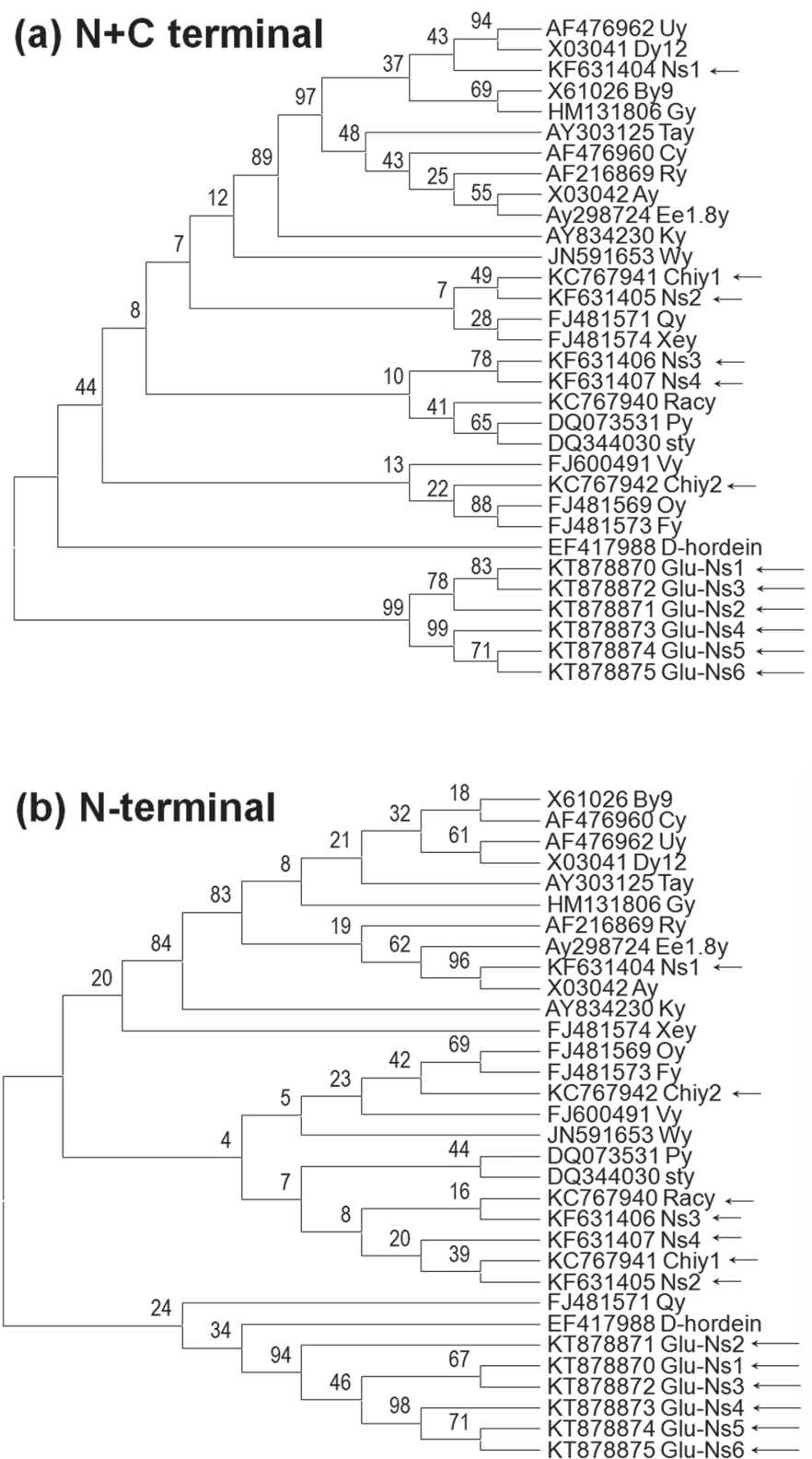

Figure 2. 


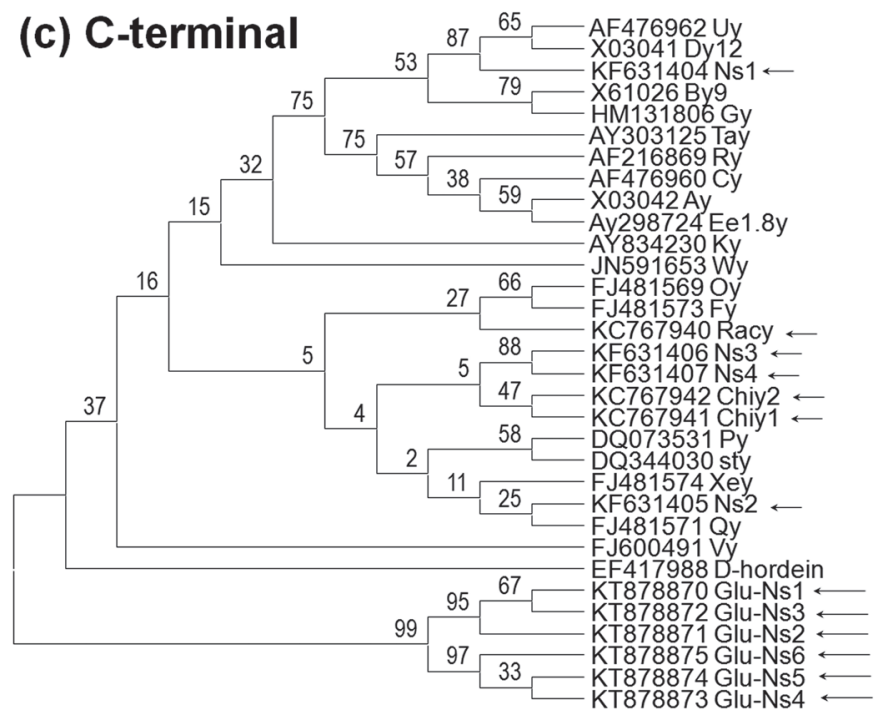

Figure 2. Phylogenetic tree of HMW-GSs from $P$. juncea and other Triticeae species with amino acid residues of N- and C-termini (a), N-termini (b), and C-termini (c). Long arrowheads indicate the P. juncea HMW-GSs in this study, and the short arrowheads indicate those of the P. juncea HMW-GSs from Kong et al. (2014) and tetraploid Leymus species (Sun et al. 2014)

close relationship with D-hordein. However, the previously published Glu-Ns (Kong et al. 2014) clustered together with other HMW-GSs. The four previously reported Glu-Ns (Ns1-Ns4) showed close relationships with the HMW-GSs from wheat and some wheat wild relatives, including Aegilops umbellulata (Uy) and two tetraploid Leymus species L. racemosus ssp. racemosus (Racy), and L. chinensis (Chiy1 and Chiy2), but were distantly related to D-hordein (Fig. 2a).

Similar tendencies for the Glu-Ns in the current and previous studies were reflected in the N- (Fig. 2b) and C-terminal trees (Fig. 2c). In both trees, all of the HMW-GSs were assembled into two major branches. One branch was formed by six current Glu-Ns sequences and showed close relationships with D-hordein, and the other branch contained the HMW-GSs from previously reported Glu-Ns and other species. Although the former (Ns1-Ns4) clustered with the HMW-GSs of different wild wheat species in the N(Fig. 2b) and C-terminal trees (Fig. 2c), their relationship with D-hordein was distant.

\section{Discussion}

We analyzed the HMW-GSs and their sequences from an Ns genome diploid species, $P$. juncea. SDS-PAGE analysis indicated that the $P$. juncea HMW-GSs were heterozygous either among or within accessions. The protein bands, which showed similar electrophoretic mobilities as subunits 7, 2, and 2.2 of the wheat references CS and Sh, were considered HMW-GSs of P. juncea (Fig. S2). Theoretically, diploid P. juncea should have had 
at the most four HMW-GSs in each seed. We found a few seeds that had more than four putative HMW-GSs. The possible contamination with proteins other than HMW-GSs or the degradation of HMW-GSs could not be excluded because no special analyses were performed, such as selective precipitation for HMW-GSs (Mackie et al. 1996).

In this study, the direct isolation of unknown P. juncea HMW-GSs failed using PCR primers that are effective in Triticeae grasses (Wang et al. 2012; Sun et al. 2014), including the previously studied $P$. juncea (Kong et al. 2014). However, isolation was successful using 5'-promotor sequence-based TAIL-PCR primers. The sequencing of 5'-promotor sequences revealed that some bases differed from known HMW-GSs at the beginning of ORFs in $P$. juncea. Some of these mutations may have led to the inability to isolate HMWGSs from P. juncea (Yang et al. 2010). We overcame this obstacle using 5'-promotor sequence-based TAIL-PCR primers and TAIL-PCR technology (Liu and Cheng 2007).

Our study is the first known attempt to isolate new HMW-GS sequences using this PCR technology. Sequencing results indicated the presence of an abnormal C-terminus (Fig. 1a). A new C-terminal primer, PPORR, was designed and used in combination with PPORF to directly amplify the ORFs. We obtained six ORFs, Glu-Ns1 to -Ns6. These ORFs varied from 1,317 to $1,323 \mathrm{bp}$ in length. However, they were not expressed as normal HMW-GS proteins, because premature stop codons occurred either in the C-termini or central repetitive domains (except for KT878871-Glu-Ns2 from PI 315080). The premature stop codon (s), which was caused by a shift mutation through a 1-2 base(s) deletion or point mutation in the triplet codons, interrupted the ORFs of HMW-GS genes from wheat and other Triticeae species and silenced these genes (Yuan et al. 2009). However, more premature stop codons occurred in the repetitive domain and a few in the C-terminus because more glutamine existed in the repetitive domain, and the substitutions of $\mathrm{C}$ to $\mathrm{T}$ in the first position of the triplet glutamine code (CAA, and $\mathrm{CAG}$ ) lead to premature stop codons TAA or TAG. The in-frame stop codons at the distal C-termini of the six Glu$N s$ were mainly responsible for gene silencing, even when no stop codon was present in the repetitive domain (Fig. S5a in KT878871_Glu-Ns2 from PI315080). A single base transition from $\mathrm{G}$ to $\mathrm{A}$ in the first position of the triplet codon GAG led to the in-frame stop codon TAG in the $\mathrm{C}$-terminus.

Although these ORFs are not likely expressed as normal HMW-GS proteins, novel modifications in the gene structures require special attention. Kong et al. (2014) also reported Glu-Ns from P. juncea. These Glu-Ns shared normal, standard y-type domain lengths and nucleotide compositions in the conserved $\mathrm{N}$ - and $\mathrm{C}$-terminal domains, as well as standard motif units in the central repetitive domain (Kong et al. 2014). However, the current Glu-Ns showed a greater similarity to D-hordein than to the Glu-Ns published by Kong et al. (2014). The Glu-Ns in our study had the longest N-terminal length (120 residues), and D-hordein had the second longest (110 residues), among all of the HMW-GS reported to date, which varied from 70-110 residues for y-types and 74-88 residues for x-types (Gu et al. 2003; Shewry et al. 2003; Pistón et al. 2007). A large DNA fragment insertion of $36 \mathrm{bp}$ that encoded the short H/YNRLNLQSIEIG peptide was responsible for the extra-long N-terminal length of Glu-Ns compared with the other HMW-GSs, with the exception of D-hordein (Fig. S5b). Small DNA fragment insertions of 3-9 bp at five dif- 
ferent positions in Glu-Ns were responsible for the longer N-terminal length compared with D-hordein. In the central repetitive domain, the Glu-Ns reported in a previous study (Kong et al. 2014) shared normal standard y-type hex- (PGQGQQ) and nonapeptide (GYYPTSPQQ) motif units (Shewry et al. 2003). The repeat motif units of the current $G l u-N s$ were unique. We observed three different motif units of 13-residue $(\mathrm{GYWH}(/ \mathrm{I} / \mathrm{Y})$ YT(/Q)S(/T)VTSPQQ), hexapeptide (consensus PGQGQQ), and tetrapeptide (ITVS). The tetrapeptide (ITVS) was shared by D-hordein and the current Glu-Ns (Gu et al. 2003; Pistón et al. 2007). However, the 13-residue repeats [GYWH (/I/Y)YT(/Q)S(/T)VTSPQQ] were restricted to the current Glu-Ns. In addition to the N-terminus and central repetitive domain, the residues at the beginning and the ending of $\mathrm{C}$ terminus were also unique. Standard y-types contained SPYHVSA and GGALSASQ, whereas the current $G l u-N s$ contained II (V) IS/FPHQ and AMLANK.

Because the Glu-Ns obtained in the current study were small (about $1.3 \mathrm{~kb}$ in ORF length) and not expressed as normal proteins, the actual genes that expressed the $P$. juncea HMW-GSs (Fig. S2, with similar electrophoretic mobilities as subunits 7, 2, and 2.2 of the wheat references) remain unknown and should be studied further. The ORF lengths of subunits 7, 2, and 2.2 were 2,373 bp (Anderson and Greene 1989), 2,520 bp (Sugiyama et al. 1985), and 2,919 bp (Wan et al. 2005), respectively. The ORF lengths of normal P. juncea HMW-GS proteins were at least $2.3 \mathrm{~kb}$. Therefore, it is important to isolate and sequence the genes encoded in these subunits to attempt to further study their gene structures.

The current Glu-Ns information is valuable for understanding evolutionary relationships among the HMW-GSs of Triticeae. The phylogenetic trees based on the residues of N- and C-termini (Fig. 2a), N-termini (Fig. 2b), and C-termini (Fig. 2c) exhibited different origins from that of the previously published Glu-Ns. The current Glu-Ns formed a separate branch located at the basal part of the tree by very high bootstrap values and were more closely related to D-hordein than the previously published Glu-Ns. However, the latter (Kong et al. 2014) had different origins from other HMW-GSs and were more related to the y-types of some wheat relatives (for example, tetraploid Leymus species and Ae. umbellulata) than D-hordein. As expected, the Glu-Ns from the previous study had a closer relationship with tetraploid Leymus species $(2 n=4 x=28$, NsNsXmXm), which shared the same Ns genome as diploid $P$. juncea and another unknown Xm genome, than the current Glu-Ns.

Our study is the first known attempt that used sequence-based TAIL-PCR to successfully isolate new HMW-GS sequences from $P$. juncea. As a result, we developed a novel method to determine the gene structures. The Glu-Ns in our study were similar to Dhordein, yet possibly more primitive than D-hordein. These results also indicated that Psathyrostachys HMW-GSs played an important role in the origin and evolution of HMW-GS among the Triticeae species. Finally, this study demonstrated an alternative approach for obtaining unknown HMW-GS sequences. 


\section{Acknowledgements}

The authors acknowledge financial support from the National Natural Science Foundation of China (U1403185) and the Key Fund Project of the Sichuan Provincial Department of Education (15ZA0021).

\section{References}

Anderson, O.D., Greene, F.C. 1989. The characterization and comparative analysis of high molecular weight glutenin genes from genomes A and B of a hexaploid bread wheat. Theor. Appl. Genet. 77:689-700.

Cao, Z., Deng, Z., Wang, M., Wang, X., Jing, J., Zhang, X., Shang, H., Li, Z. 2008. Inheritance and molecular mapping of an alien stripe-rust resistance gene from a wheat-Psathyrostachys huashanica translocation line. Plant Sci. 174:544-549.

Garg, M., Tanaka, H., Tsujimoto, H. 2009. Exploration of Triticeae seed storage proteins for improvement of wheat end product quality. Breed. Sci. 59:519-528.

Gu, Y.Q., Anderson, O.D., Londeorë, C.F., Kong, X.Y., Chibbar, R.N., Lazo, G.R. 2003. Structural organization of the barley D-hordein locus in comparison with its orthologous regions of wheat genomes. Genome 46:1084-1097.

Hsiao, C., Wang, R.R-C., Dewey, D.R. 1986. Karyotype analyses and genome relationships of 22 diploid species in the tribe Triticeae. Can. J. Genet. Cytol. 28:109-120.

Kong, L.N., Liang, Y., Qin, L.M., Sun, L., Xia, G.M., Liu, S.W. 2014. Characterization of high molecular weight glutenin subunit genes from the Ns genome of Psathyrostachys juncea. Dev. Genes Evol. 224:189196.

Liu, Y.G., Cheng, Y.L. 2007. High-efficiency thermal asymmetric interlaced PCR for amplification of unknown flanking sequences. Biotechniques 43:649-656.

Mackie, A.M., Lagudah, E.S., Sharp, P.J., Lafiandra, D. 1996. Molecular and biochemical characterisation of HMW glutenin subunits from T. tauschii and the D genome of hexaploid wheat. J. Cereal Sci. 23:213-225.

Mujeeb-Kazi, A., Cortes, A., Riera-Lizarazu, O. 1995. The cytogenetics of a Triticum turgidum $\times$ Psathyrostachys juncea hybrid and its backcross derivatives. Theor. Appl. Genet. 90:430-437.

Pistón, F., Shewry, P.R., Barro, F. 2007. D hordeins of Hordeum chilense: a novel source of variation for improvement of wheat. Theor. Appl. Genet. 115:77-86.

Plourde, A., Fedak, G., St-Pierre, C.A., Comeau, A. 1990. A novel intergeneric hybrid in the Triticeae: Triticum aestivum $\times$ Psathyrostachys juncea. Theor. Appl. Genet. 79:45-48.

Rasheed, A., Xia, X.C., Yan, Y.M., Appels, R., Mahmood, T., He, Z.H. 2014. Wheat seed storage proteins: Advances in molecular genetics, diversity and breeding applications. J. Cereal Sci. 60:11-24.

Shewry, P.R., Halford, N.G., Tatham, A.S., Popineau, Y., Lafiandra, D., Belton, P.S. 2003. The high molecular weight subunits of wheat glutenin and their role in determining wheat processing properties. Adv. Food Nutr. Res. 45:219-302.

Sugiyama, T., Rafalski, A., Peterson, D., Soll, D. 1985. A wheat HMW glutenin subunit gene reveals a highly repeated structure. Nucleic Acids Res. 13:8729-8737.

Sun, Y.Q., Pu, Z.J., Dai, S.F., Pu, X.X., Liu, D.C., Wu, B.H., Lan, X.J., Wei, Y.M., Zheng, Y.L., Yan, Z.H. 2014. Characterization of y-type high-molecular-weight glutenins in tetraploid species of Leymus. Dev. Genes Evol. 224:57-64.

Tamura, K., Stecher, G., Peterson, D., Filipski, A., Kumar, S. 2013. MEGA6: Molecular evolutionary genetics analysis version 6.0. Mol. Biol. Evol. 30:2725-2729.

Wan, Y.F., Yan, Z.H., Liu, K.F., Zheng, Y.L., D’ Ovidio, R., Shewry, P.R., Halford, N.G., Wang, D.W. 2005. Comparative analysis of the D genome-encoded high-molecular weight subunits of glutenin. Theor. Appl. Genet. 111:1183-1190.

Wang, X.Q., Yan, Z.H., Dai, S.F., Liu, D.C., Wei, Y.M., Zheng, Y.L. 2012. Molecular characterization of four HMW glutenin genes from Heteranthelium piliferum C. E. Hubbard and Henrardia persica (Banks et Solander) Hochstetter. Genet. Resour. Crop Evol. 59:1309-1318. 
Yang, F., Yan, Z.H., Dai, S.F., Han, C., Hu, X.G. 2010. Isolation and characterization of upstream sequences of two high molecular weight glutenin subunit genes from Psathyrostachys juncea. J. Sichuan Agricultural University 28:397-402.

Yuan, Z.W., Chen, Q.J., Zhang, L.Q., Yan, Z.H., Zheng, Y.L., Liu, D.C. 2009. Molecular characterization of two silenced y-type genes for Glu-B1 in Triticum aestivum ssp. yunnanese and ssp. tibetanum. J. Integr. Plant Biol. 51:93-99.

\section{Electronic Supplementary Material (ESM)}

Electronic Supplementary Material (ESM) associated with this article can be found at the website of CRC at http://www.akademiai.com/content/120427/

Electronic Supplementary Table S1. PCR primer sequence

Electronic Supplementary Table S2. PCR components and conditions

Electronic Supplementary Figure S1. Description of PCR primers used in this study

Electronic Supplementary Figure S2. SDS-PAGE analysis of the HMW-GSs from four P. juncea accessions. CS (Chinese Spring and Sh (Shinchunaga) are two wheat cultivars with known HMW-GSs; all others are P. juncea accessions

Electronic Supplementary Figure S3. PCR amplification for promoter sequences (a), ORFs of HMW-GS in preamplification (b), primary TAIL-PCR (c) and secondary TAIL-PCR (d) and in complete ORFs (e) from P. juncea accessions. Lanes 1-5 are accessions PI 75737, PI 315080, PI 531826, PI 619483 and PI 565065, respectively

Electronic Supplementary Figure S4. Alignment of HMW-GS gene promoter sequences among from five P. juncea accessions. Other HMW-GSs including y-type representatives from species with A, B, C, D, F, G, O, P, Q, R, St, Ta, U, W, and Xe genomes from the GenBank accessions X 03042, X 61026, AF 476960, X 03041, Ay 298724, FJ 481573, HM 131806, FJ481569, Py DQ 073531, FJ 481571, AF 216869, DQ 344030, AY 303125, AF 476962, JN 591653, and FJ 481574, respectively

Electronic Supplementary Figure S5. Comparison of HMW-GS genes from P. juncea with orthologous sequences from other Triticeae species in repetitive (a), and $\mathrm{N}$ - and $\mathrm{C}$ - terminal (b) domains. Other HMW-GSs including y-type representatives from species with A, B, C, D, F, G, O, P, Q, R, St, Ta, U, W, and Xe genomes from the GenBank accessions X 03042, X 61026, AF 476960, X 03041, Ay 298724, FJ 481573, HM 131806, FJ481569, Py DQ 073531, FJ 481571, AF 216869, DQ 344030, AY 303125, AF 476962, JN 591653, and FJ 481574, respectively 